\title{
BMJ Open Hyaluronic Acid Binding Sperm Selection for assisted reproduction treatment (HABSelect): study protocol for a multicentre randomised controlled
} trial

\author{
K D Witt, ${ }^{1}$ L Beresford, ${ }^{1}$ S Bhattacharya,${ }^{2} \mathrm{~K}$ Brian, ${ }^{3}$ A Coomarasamy, ${ }^{4} \mathrm{R}$ Hooper, ${ }^{1}$ \\ J Kirkman-Brown, ${ }^{4}$ Y Khalaf, ${ }^{5}$ S E Lewis, ${ }^{6}$ A Pacey, ${ }^{7}$ S Pavitt, ${ }^{8}$ R West, ${ }^{9}$ D Miller ${ }^{10}$
}

To cite: Witt KD, Beresford L, Bhattacharya $\mathrm{S}$, et al. Hyaluronic Acid Binding Sperm Selection for assisted reproduction treatment (HABSelect): study protocol for a multicentre randomised controlled trial. BMJ Open 2016:6:e012609. doi:10.1136/bmjopen-2016012609

- Prepublication history and additional material is available. To view please visit the journal (http://dx.doi.org/ 10.1136/bmjopen-2016012609).

Received 11 May 2016 Revised 19 August 2016 Accepted 9 September 2016

CrossMark

For numbered affiliations see end of article.

Correspondence to Dr D Miller;

d.miller@leeds.ac.uk

\section{ABSTRACT}

Introduction: The selection of a sperm with good genomic integrity is an important consideration for improving intracytoplasmic sperm injection (ICSI) outcome. Current convention selects sperm by vigour and morphology, but preliminary evidence suggests selection based on hyaluronic acid binding may be beneficial. The aim of the Hyaluronic Acid Binding Sperm Selection (HABSelect) trial is to determine the efficacy of hyaluronic acid (HA)-selection of sperm versus conventionally selected sperm prior to ICSI on live birth rate (LBR). The mechanistic aim is to assess whether and how the chromatin state of HA-selected sperm corresponds with clinical outcomes-clinical pregnancy rate (CPR), LBR and pregnancy loss (PL).

Methods and analysis: Couples attending UK Centres will be approached, eligibility screening performed and informed consent sought.

Randomisation will occur within 24 hours prior to ICSI treatment. Participants will be randomly allocated 1:1 to the intervention arm (physiological intracytoplasmic sperm injection, PICSI) versus the control arm using conventional methods (ICSI). The primary clinical outcome is LBR $\geq 37$ weeks' gestation with the mechanistic study determining LBR's relationship with sperm DNA integrity. Secondary outcomes will determine this for CPR and PL. Only embryologists performing the procedure will be aware of the treatment allocation. Steps will be taken to militate against biases arising from embryologists being nonblinded. Randomisation will use a minimisation algorithm to balance for key prognostic variables. The trial is powered to detect a $5 \%$ difference $(24-29 \%$ : $\mathrm{p}=0.05)$ in LBR $\geq 37$ weeks' gestation. Selected residual sperm samples will be tested by one or more assays of DNA integrity.

Ethics and dissemination: HABSelect is a UK NIHREME funded study (reg no 11/14/34; IRAS REF. 13/YH/ 0162). The trial was designed in partnership with patient and public involvement to help maximise patient benefits. Trial findings will be reported as per CONSORT

\section{Strengths and limitations of this study}

- Hyaluronic Acid Binding Sperm Selection (HABSelect) is one of the only trials with sufficient power to test the efficacy of a spermselection procedure that has shown some promise for improving live birth rate but without conclusive evidence hitherto.

- The trial has closely linked clinical and basic science aspects that makes best use of the resources provided by participating couples. Both components will advance clinical and mechanistic understanding.

- Since the intervening embryologist is aware of the arm allocation, there may be a potential for subconscious embryo selection bias, particularly in smaller clinics with fewer staff. This effect, however, should be mitigated by data capture, including details of the embryologist involved and close data monitoring by the independent steering committee.

- There are likely to be potentially confounding variations in semen quality that could affect the interpretation of clinical outcomes, but these should be mitigated by careful recording of semen profiles and their stratification according to HBA scoring. A hierarchy of sperm chromatin quality assays will allow us to minimise the effects of sample availability while maximising information content.

- Mechanistic work is entirely dependent on the efficient recovery of residual processed sperm from participating centres following treatment. The success or otherwise of this recovery process is very likely to vary among participating centres.

guidelines and will be made available in lay language via the trial web site (http://www.habselect.org.uk).

Trial registration number: ISRCTN99214271; Pre-results. 


\section{BACKGROUND}

One in seven couples experience difficulty conceiving a child and rises in the prevalence of infertility and the number of couples seeking help via assisted reproduction technologies (ARTs) is now evident. ${ }^{1}{ }^{2}$ In 2012, almost 47000 couples in the UK alone were treated with ART, comprising 62000 treatment cycles, over half of which involved intracytoplasmic sperm injection (ICSI), a technique originally developed to treat male infertility. ${ }^{2}$ Currently, live birth rates (LBRs) following ICSI treatment are at an average of $\sim 24 \%$ per treatment cycle, a rate that has remained virtually unchanged in the last 10 years. Up to $50 \%$ of infertility cases are thought to have a male factor origin ${ }^{3}$ and with ICSI fast becoming the favoured choice for fertilisation irrespective of the male factor, ${ }^{2}$ there is a more urgent need for improvements in its efficacy. To date, however, compared with egg and embryo quality, relatively little effort has been expended on improving sperm quality beyond processing semen according to WHO guidelines. ${ }^{4}$ Such processing may be less effective for ICSI where the egg itself offers no effective barrier to direct insemination by defective sperm, and sperm selection is subjectively dependent on the treating embryologist.

Sperm chromatin structure plays a vital role in protecting paternal DNA integrity by condensing the sperm DNA over 10-fold compared with somatic cell nuclei. Ordinarily, natural selection is effective at screening out defective sperm that have failed to maintain DNA integrity as they transport through the female reproductive tract. Importantly, as this 'triaging' step is omitted in the direct sperm transfer of ICSI, a greater understanding of the relationship between sperm DNA integrity (and conversely DNA fragmentation) and embryonic developmental potential is needed. Numerous studies have shown clear inverse relationships between sperm DNA fragmentation anomalies in the ejaculate and clinical pregnancy (CPR) or live birth (LBR) rates in in vitro fertilisation (IVF) ${ }^{5-10}$ However, the relationship with ICSI outcomes is less clear. We, among others, have reported that miscarriage is a risk factor in ICSI in relation to sperm DNA fragmentation, ${ }^{11}{ }^{12}$ and this may result from an oocytemediated DNA repair process ${ }^{13-16}$ that adequately supports clinical pregnancy (hence the lack of an association between DNA fragmentation and clinical pregnancy in ICSI compared with IVF), but may be inadequate to sustain it with resulting pregnancy loss (PL). There remains a need to develop more sophisticated techniques to identify functional spermatozoa from those that are immotile, have poor morphology, have poor DNA integrity or are simply incapable of fertilising oocytes. ART sperm preparation including differential density gradient centrifugation has been found to result in enrichment of sperm with intact chromatin, which in turn is likely to improve the chances of a successful clinical outcome. ${ }^{17} 18$ While success rates are known to vary widely across clinics, further innovations are needed to improve the plateaued average LBR of $24 \%$ for IVF and IVF-ICSI.
Selecting sperm binding to hyaluronic acid (HA) for ICSI is thought to be one such innovation. HA is the natural, non-sulfated glycosaminoglycan secretion of the cervical mucus and the cumulus-öopherus complex. ${ }^{19}$ Sperm reaching HA-coated surfaces can bind to and potentially digest the HA, and their subsequent hyperactivation may further facilitate their reaching the egg. ${ }^{20} 21$ Immature sperm with excessive cytoplasm appear to have a lower affinity for HA and higher rates of aneuploidy and DNA fragmentation. ${ }^{22-24}$ Studies using a HA-selection procedure for ICSI reported higher numbers of grade 1 embryos following ICSI, ${ }^{25}$ an increase in clinical pregnancy rate (CPR) with a corresponding drop in miscarriage rate ${ }^{26}$ and most recently, a significant reduction in PL and a significantly improved LBR in this group. ${ }^{27}$ These outcomes, while encouraging, were drawn from relatively small sample sizes that were insufficiently powered to conclusively test the efficacy of sperm selection by HA-binding for ICSI. ${ }^{28} 29$

\section{HYPOTHESIS}

The Hyaluronic Acid Binding Sperm Selection (HABSelect) trial is designed to test the hypothesis that selection of sperm for injection using HA binding prior to ICSI has beneficial effects on clinical outcomes compared with standard ICSI. The trial's main strength is its accommodation of clinical and basic science aspects that are fully complementary. Its parallel, mechanistic investigations will allow us to determine whether HA-binding mitigates for potentially genotoxic levels of DNA fragmentation in patients' sperm.

\section{METHODS AND ANALYSIS}

\section{Study design and objectives}

HABSelect is of a phase III, two arm, multicentre, blinded, efficacy clinical trial with mechanistic evaluation. The primary objective of the clinical trial is to determine the efficacy of HA-selected intracytoplasmic sperm injection (physiological intracytoplasmic sperm injection, PICSI) versus conventional ICSI where the primary outcome measure will be LBR $\geq 37$ weeks' gestation. The primary mechanistic objective is to evaluate whether HA-selection can compensate for poor sperm quality and investigate HA-binding score (HBS) in relation to chromatin integrity and LBR.

Secondary objectives will include a determination of the impact of the intervention on CPR based on detection of fetal heartbeat and/or fetal sac at 6-9 weeks' gestation and miscarriage rate defined as PL after confirmation of clinical pregnancy. The study design is detailed in the consort diagram (figure 1).

\section{Eligibility and recruitment}

HABSelect participant couples will recruit from multiple assisted conception units across England and Scotland. All participating sites will be recognised teaching institutions (or equivalent) accredited in the performance of 


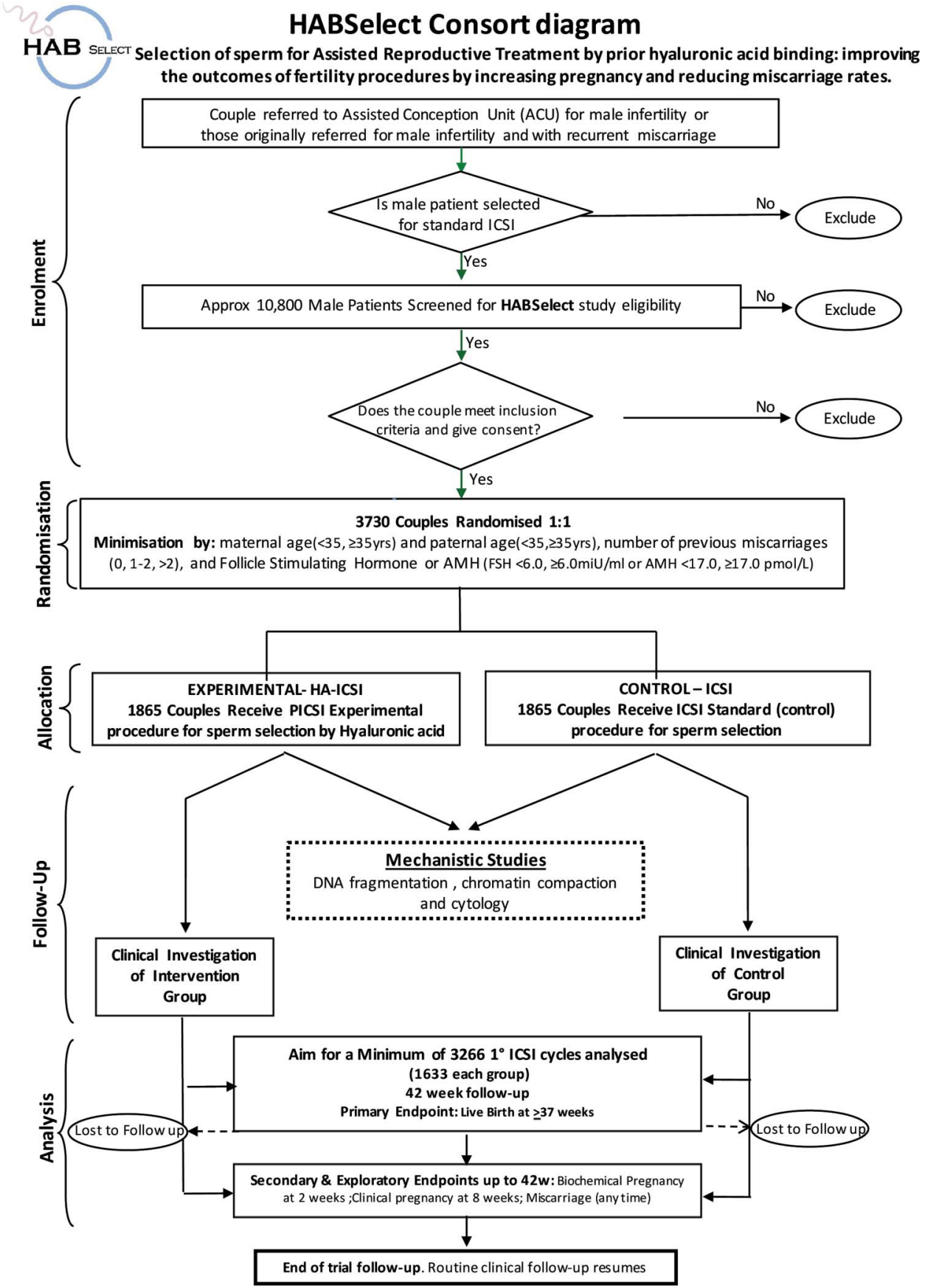

Figure 1 HABSelect consort chart. The chart was designed according to CONSORT guidelines (http://www.consort-statement. org) and shows the main aspects of the clinical trial and its relationship with clinical and mechanistic outcomes and reporting. HA, hyaluronic acid; HABSelect, Hyaluronic Acid Binding Sperm Selection; ICSI, intracytoplasmic sperm injection; PICSI, physiological intracytoplasmic sperm injection.

ICSI fertility treatments and have been initially selected on the basis of potentially high recruiting capabilities using records held by the Human Fertilisation \& Embryology Authority (HFEA). Sites commonly have a mix of NHS and private facilities treating publicly funded and fee-paying patients. Ethical approvals will include recruitment not limited to couples receiving NHS reimbursed treatment. To facilitate and assist in achieving recruitment targets, four clinical advisors will be appointed who will oversee their own centres and those in their adjacent regional areas. They will be supported by the National Institute for Health Research (NIHR) Clinical Research Network, which collects recruitment data on a monthly basis. All issues arising during the conduct of the trial will be discussed in regular monthly management meetings and any unresolved issues referred to one of two independent trial overseeing committees. Couples will be identified as candidates for the HABSelect study by local clinical or research staff if they have opted for or been advised to make use of ICSI-based procedures. Normally, 
routine WHO-based assessment of ejaculate quality is sufficient for men to be selected for ICSI procedures over IVF.

The clinical team will check that the couple meets the inclusion and exclusion criteria (box 1), and only couples meeting these criteria will be approached to provide consent to participate. Screening, confirmation of eligibility and formal enrolment onto the study will be followed by the completion of baseline assessments, and the couple will enter the ICSI clinical care pathway. The female participant will then start a follicle stimulation regimen according to the treatment centres' locally approved protocol.

\section{Randomisation}

A 1:1 randomisation of 'experimental' of HA-ICSI using the physiological intracytoplasmic sperm injection (PICSI) sperm-selection dish (PICSI) versus 'control' standard vigour with morphology ICSI sperm selection (ICSI) where the inclusion of polyvinylpyrollidone slows the sperm down sufficiently for pipette capture. Randomisation will take place within 24 hours prior to the insemination and will be performed by an authorised member of staff at the centre (typically the embryologist), using a custom, web-based 24-hour automated randomisation system employing a computer-generated minimisation algorithm according to maternal age $(<35, \geq 35)$, paternal age $(<35, \geq 35)$, number of previous miscarriages $(0,1-2,>2)$ and hormonal indicators of ovarian reserve used currently in the participating clinics (follicle stimulating hormone (FSH) $<6.0, \geq 6.0 \mathrm{miU} / \mathrm{mL}$ or anti-müllerian hormone (AMH) $<17.0, \geq 17.0 \mathrm{pmol} / \mathrm{L}$ when FSH is not available). Minimisation will not include HBS. Minimisation factors will be balanced separately

\section{Box 1 Study eligibility criteria}

\section{Inclusion criteria}

1. Couples able to provide informed consent.

2. Couples undergoing ICSI procedure.

3. Female:

A. Age: $18-43$.

B. Body mass index: $19.0-35.0 \mathrm{~kg} / \mathrm{m}^{2}$

C. FSH level 3.0-20.0 miU/mL and/or $A M H \geq 1.5 \mathrm{pmol} / \mathrm{L}$.

4. Male:

A. Age: 18-55.

B. Able to produce freshly ejaculated sperm for the treatment cycle.

Exclusion criteria

1. Couples who have not consented prior to ICSI will be ineligible.

2. Couples using non-ejaculated sperm.

3. Couples using donor gametes.

4. Men with vasectomy reversal; cancer treatment involving any chemotherapy and/or radiotherapy in the past 2 years.

5. Previous participation in the HABSelect trial.

6. Split IVF/ICSI procedures.

7. If $\mathrm{FSH}$ and $\mathrm{AMH}$ are tested and either measure falls outside the accepted range. within each site. Research nurses and treating clinicians including principal investigators will be blinded to arm allocation.

\section{Withdrawal criteria}

Participants can withdraw at any time prior to egg collection or where, in the opinion of the investigator or the care providing clinician or clinical team, it is medically necessary to do so. Study personnel will make every effort to obtain and record information about the reasons for discontinuation, any adverse events and to follow-up the women for all safety and efficacy outcomes, as appropriate. A clear distinction will be made as to whether the patient is withdrawing from trial treatments/procedures while allowing further follow-up, or whether the patient refuses any follow-up. If a patient explicitly withdraws consent to have any data recorded, their decision will be respected and recorded on the electronic data capture system. All communication surrounding the withdrawal will be noted in the patient's records, and no further case report forms (CRFs) will be completed for that patient.

\section{Trial intervention and participant follow-up}

The HA-ICSI intervention will use the Conformité Européenne (CE) marked and Medicines and Healthcare Products Regulatory Agency (MHRA) approved PICSI dish (Sterling Scientific, USA) alongside their Hydak HBS slide. These products offered the best prospect for assuring continuity of supply, quality control and relative ease of use. (figure 2). An alternative medically approved product (Sperm-slow) was considered by the clinical advisory teams on the evidence that it and PICSI have similar efficacy ${ }^{30}$ but was rejected in favour of PICSI, which has been more widely reported in the context of CPR and miscarriage. ${ }^{26}{ }^{27} \mathrm{HBS}$ will be obtained from both arms of the trial, but only the HA-ICSI arm will make use of the PICSI plates. There are no other planned interventions in the study. All protocol-required assessments and data collection will be recorded on trial-specific CRFs at each site. Any remaining sperm sample will be processed and frozen stored (four equal aliquots per sample) according to the requirements of the mechanistic research evaluation. The trial will also make use of the approved tissue bank to facilitate transfer of the samples between participating sites and the mechanistic research laboratories.

Following ICSI, couples will resume standard care with no further scheduled trial-specific follow-up. However, the couples participating in the HABSelect trial will have their unique ID number allocated on enrolment to the study and linked to the female partner's patient record so that routine fetal/pregnancy outcome data can be captured and recorded on the web-based database following the template required by the HFEA. No patient identifiable information will be entered. All data sharing 


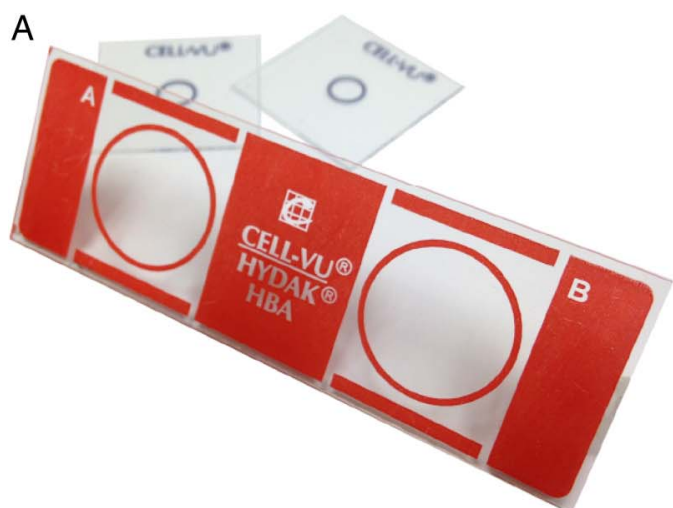

B

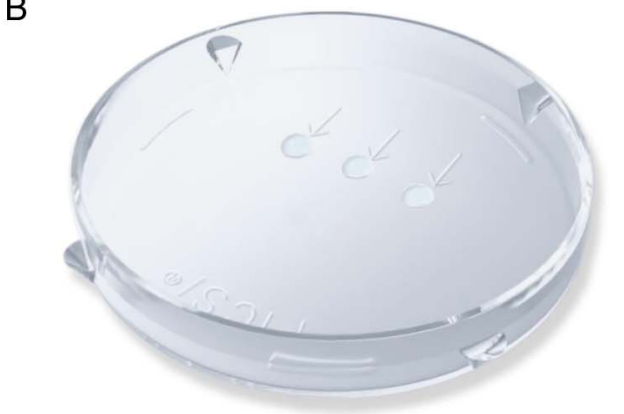

C

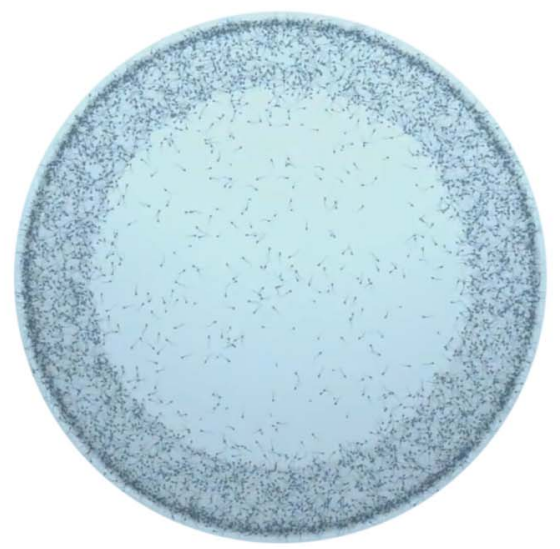

Figure 2 (A) Hydak slide showing twin chambers used to obtain sample \% HBS. (B) PICSI plate showing channels (arrows) into which sperm suspensions are introduced. Mature, motile sperm migrate towards the hyaluronic coated dots at one end of each channel where they bind.

(C) Photomicrograph from time-lapse recording showing a single PICSI hyaluronan dot. Note accumulated sperm on periphery, considered to be moribund. Tethered, motile sperm for ICSI are selected from the more sparsely populated central region. Reproduced from Biocoat original datasheets with permission. HBS, HA-binding scores; PICSI, physiological intracytoplasmic sperm injection.

between clinical and mechanistic arms of the study will follow principles of good Information Governance (www http://systems.hscic.gov.uk/infogov).

\section{Mechanistic study}

A key aspect of HABSelect is the recovery of residual pelleted sperm for assessment of male patients' sperm genomic integrity based on measures of DNA fragmentation and chromatin compaction or condensation state (TUNEL, Comet, HALO, Acridine Orange, Aniline Blue and chromomycin A3 staining; see figure $3 \mathrm{~A})$. Use of several independent measures of paternal genomic integrity will address the issue of assay variability. $^{78} 81-36$ This strategy will help maximise the information we obtain from these samples and assist interpretation of data arising from past and future studies that make use of any of these assays.

Samples will be selected initially on whether a clinical pregnancy was reported or not (blinded to arm allocation but balanced numbers), and the process reiterated until 1200 samples have been tested with two or more of the same assays across three $(<50 \% 50-65 \%,>65 \%)$ sperm HBS strata. The precise number of assays will depend on sample quality, as measured by cytology, based mainly on the number of recovered sperm. A hierarchy of assays (figure 3A) also takes account of limited sperm numbers following cytological inspection that may be encountered in any sample, while making provision for maximising information content. In addition to cytology, should only two tests be possible, the hierarchy will always include one assay of DNA fragmentation (Comet or TUNEL) and one assay of chromatin compaction (Aniline Blue or CMA3). HALO overlaps both variables and will be used where only one assay is possible. The mechanistic sample size is based on the structural equation modelling (SEM) linking the clinical and mechanistic outcomes ('Statistical considerations' section).

\section{Statistical considerations}

\section{Clinical sample size and estimates}

We estimate from HFEA audit data ${ }^{2}$ that $\sim 6000$ couples per annum undergoing ICSI will be eligible across all centres. Assuming a LBR following ICSI of $\sim 24 \%,{ }^{34}$ a minimum of 3266 couples will be needed to detect a $5 \%$ improvement in LBR (25-29\%) at $\geq 37$ weeks with $90 \%$ power. A $10 \%$ loss to follow-up is accommodated although it is anticipated that compliance will be high given the lateness of randomisation and the routine nature of collecting data on biochemical pregnancy (BP), clinical pregnancy (CP) and live birth (LB) data in this population.

\section{Clinical statistical analysis}

Our unit of analysis will be the couple. Baseline characteristics will be tabulated. We have elected to focus on the outcome of the first fresh ICSI cycle in each randomised couple and powered the trial accordingly. The analysis will be by intention to treat. Numbers of couples at different stages of the trial are summarised in the CONSORT diagram (figure 1).

The primary outcome is the proportion of women who experience a live birth $\geq 37$ weeks. Secondary outcomes are the respective proportions of women who experience the following: a clinical pregnancy (presence of a fetal heartbeat or fetal sac at 6-9 weeks' gestation); 
a PL; a live birth <37 weeks. Outcomes in experimental and control arms will be compared using multivariable logistic regression, adjusting for centre and for factors used in the minimisation, with effects summarised as ORs with 95\% CIs. If there is evidence that the CPR differs between the trial arms, then secondary analyses will be carried out taking only women with a clinical pregnancy as the denominator. In all cases, results of primary analyses will be given more weight than those of the secondary analyses.

Every attempt will be made to gather data on all women randomised, irrespective of compliance with the treatment protocol. If baseline assessments of covariates are missing, we will use mean values or missing value indicators to replace them. ${ }^{37}$ If any outcome data are missing, we will analyse only those with outcome data, adjusting for baseline covariates. This approach is unbiased if reasons for the outcome being missing can be related to observed covariates (the so-called 'missing at random' assumption) ${ }^{36}$ If the primary outcome is missing for $>5 \%$ of couples, then a sensitivity analysis will be conducted to explore the 'missing at random' assumption, using a pattern mixture approach. $^{38}$

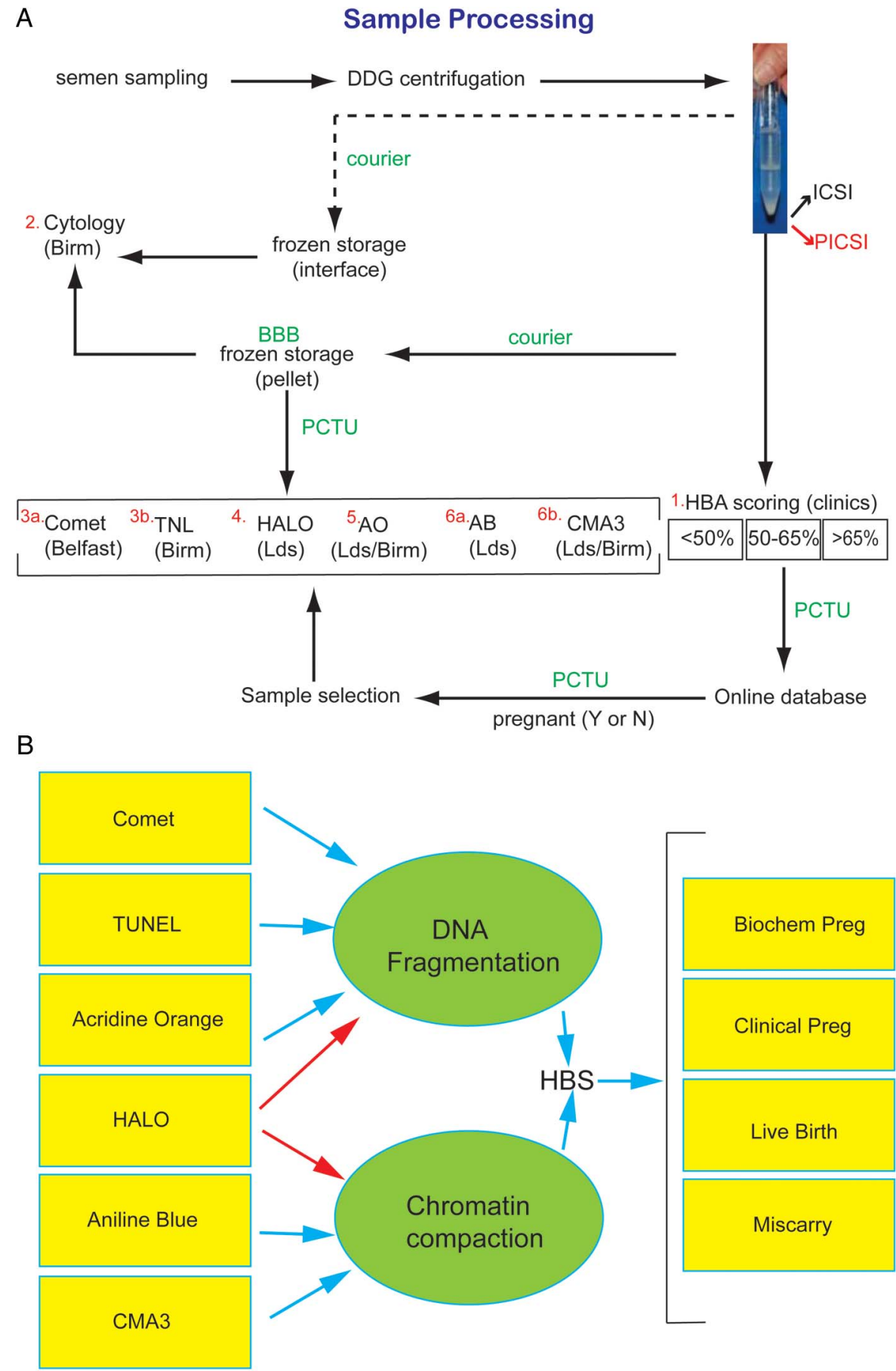


Exploratory analyses will investigate possible modifiers of the treatment effect, including the factors used for minimisation as well as sperm concentration $\left(<15 \times 10^{6}\right.$ vs $\left.\geq 15 \times 10^{6}\right)$ and hyaluronan binding score $(>65 \%$ vs $\leq 65 \%$ ). Depending on numbers available, we may also compare very low $(\leq 25 \%)$ and low $(>25 \%, \leq 65 \%)$ HBS subgroups. In each case, an interaction test will first be used to determine whether there is a basis for investigating treatment efficacy within subgroups. Subgroup analyses will be hypothesis generating only, and results will be treated with caution.

As the two arms of this study are compatible with the equivalent arms of the recent US NIH trial ${ }^{26}$ they can be included in any future meta-analysis of the data.

\section{Mechanistic sample size and estimates}

The mechanistic evaluation will be conducted through a Structural Equation Modeling (SEM) approach that is particularly well suited to estimating causal relationships using a combination of quantitative data and qualitative causal assumptions. ${ }^{39} 40$ DNA fragmentation will be measured by the comet and acridine orange assays and summarised through the latent variable DNA fragmentation (figure 3A). Similarly, chromatin compaction measured by aniline blue and CMA3 assays will be summarised through the latent variable, chromatin compaction. HALO provides a separate covariate of sperm nuclear integrity that contributes to both latent variables. These two latent variables will then be regarded as covariates in a regression model for HBS, which in turn is a covariate for the logistic regressions for each of the primary and secondary clinical outcomes. This model is represented in figure $3 \mathrm{~B}$ for clarity. Other models where the two latent variables are also covariates in the logistic regressions for the outcomes will be considered where sufficient samples are available for robust estimation.

Evidence from blinded data suggests that $70 \%$ of samples will permit two or more tests and therefore some measure of both. On the basis of existing reports, ${ }^{26} 27{ }^{41}$ the benefit of HA-ICSI may be greatest for couples with the lowest HBS and the relationships between HBS and the key outcomes will be non-linear. Hence, interpretation of results of SEM could be compromised without considering a balanced HBS stratification of the samples. The proportion of samples in the lowest stratum (estimated at $14 \%$ of all samples) is our most constraining variable and has the strongest influence on the final sample size. Our current estimate for the LBR within the lowest HBS stratum is $18 \%$. Robust estimates of the coefficients within a logistic regression have been thoroughly explored in simulation studies by Peduzzi et $a l,{ }^{42}$ where it was established that 10 events per covariate are required. Consequently, within this lowest HBS stratum we might expect 31 live births. The Peduzzi rule ${ }^{42}$ requires at least 30 live births to robustly estimate the coefficients of three covariates; one for HBS the variable of interest, one for treatment (ICSI/ PICSI) and one for the Nelson-Lawlor log odds of live birth. ${ }^{43}$ These covariates are omitted from figure $3 \mathrm{~B}$ for clarity. Working back, we estimate that 1216 samples balanced across all strata will be required to record at least 30 live births in the lowest HBS stratum. This figure is approximately half of all finally stored samples, giving ample room for adjustment. Structural equation modelling will be undertaken with the software MPLUS V.7.4.

Figure 3 (A) Flow chart for mechanistic sample processing. (B) Structural equation modelling for mechanistic analyses. (A) Residual sperm samples will be scored (HBS) before freezing and shipping to central storage (Birmingham BioBank; BBB). The clinical trials unit (Pragmatic Clinical Trials Unit; PCTU) will select samples for analysis based on their HBS stratification (1) and whether the sample is from a couple who achieved or did not achieve a clinical pregnancy (arm allocation blinded to the investigating team). Those samples will then be assessed for further analyses following standard cytological evaluation (2). As many tests of DNA fragmentation as possible will be carried out if sufficient sperm are available, by distributing (frozen) sample aliquots between the three mechanistic study centres (Birmingham, Belfast and Leeds). Hierarchical priority of testing will be Comet $(3 a)=$ TUNEL $(3 b)>$ HALO $(4)=A O(5)>A B(6 a)=C M A 3(6 b)$. If sample is limiting, the revised testing priority requires at least one assay of DNA fragmentation (3a preferred) and one assay of chromatin compaction (6b preferred) to be carried out. If there is only sufficient sperm for one assay, then priority will be given to HALO (4) as its measure of DNA fragmentation is closely dependent on chromatin compaction. These priorities may change as more data become available. (B) In brief, the Comet, TUNEL and Acridine Orange assays measure sperm DNA fragmentation. The CMA3 and Aniline Blue assays measure sperm chromatin compaction. HALO is a measure of DNA fragmentation and chromatin compaction. These latent variables will be regarded as covariates in a regression model for HBS, which in turn is a covariate for the logistic regressions for each of the primary and secondary clinical outcomes. The HBS will vary in relation to DNA fragmentation and chromatin compaction and will then predict the key outcomes. Omitted from the diagram are other factors in this relationship that also influence outcomes, namely the treatment given (ICSI/PICSI) and the couples concerned: Nelson and Lawler provide details of nine factors, some of which are non-linear, but for which the chances of outcomes can be derived with an online routine (http://www.ivfpredict.com). We propose to use the Nelson-Lawlor log odds as a single predictor variable rather than the nine factors. Note that we anticipate the relationships between HBS, other factors, treatment and the key outcomes to be complex: potentially non-linear. To explore this possibility, the samples will be stratified by HBS as follows: $<50 \%, 50-65 \%$ and $>65 \%(A)$. Note that for clarity, the path diagram (B) focuses on only those variables specific to the mechanistic work. HBS, hyaluronan binding score; ICSI, intracytoplasmic sperm injection; PICSI, physiological intracytoplasmic sperm injection. 


\section{End point analyses}

Primary end point analysis

The primary end point is the proportion of women randomised who experience a live birth of $\geq 37$ weeks. This proportion has as its denominator the number of women who are followed up after their ICSI cycle following fresh embryo transfer (UK law permits up to three) postrandomisation per arm. Its numerator is the number of women who conceive and proceed to have a live birth of $\geq 37$ weeks. The proportion will be compared between arms using multivariable logistic regression adjusting for centre and for factors used in the minimisation. An OR with $95 \%$ CI will be calculated.

\section{Secondary end point analyses}

The secondary end points are the respective proportions of women who:

- Experience a clinical pregnancy based on the presence of fetal heartbeat or fetal sac at 6-9 weeks' gestation.

- Experience a clinical pregnancy and miscarry.

- Experience a clinical pregnancy and proceed to a live birth of $<37$ weeks.

These proportions will follow the same calculations as primary end point analysis.

\section{Subgroup analyses}

For binary outcomes, results will be expressed as OR with $95 \%$ CIs of pregnancy success in either arm. The exploratory subgroup analyses will follow minimisation criteria as described in the 'Randomisation' section above with addition of

- HBS (high $(>65 \%)$ vs low $(\leq 65 \%))$,

- Sperm concentration $\left(<15 \times 10^{6}\right.$ vs $\left.\geq 1 \times 10^{6}\right)$.

We shall also analyse a very low $(\leq 25 \%)$ versus low $(>25 \%, \leq 65 \%)$ HBS subgroups. A more detailed clinical statistical analysis plan (SAP) is provided in online supplementary appendix 1 . The more uncertain and dynamic nature of the requirements for mechanistic analyses excludes the provision of a detailed mechanistic SAP before actual data acquisition begins. Readers are referred to the 'Mechanistic sample size and estimates' section where some details of the statistical approach are provided.

\section{ETHICS AND DISSEMINATION}

As HABSelect is a fertility study, both partners will need to provide informed consent before being randomised to the study. Clinical intervention is minimal and applies to in vitro conducted process of sperm selection; similarly, the mechanistic investigation will only make use of the residual sperm left over after the treatment. Taking these facts into consideration, the sponsor has determined that no additional 'active' monitoring for patient safety and adverse event reporting is required and only related unexpected serious adverse events (RUSAE) will be reportable to ethics committees and sponsor. Every attempt will be made to collect full follow-up data on all couples, and it is anticipated that missing data will be minimal due to routine nature of collected data and its compliance with the pregnancy outcomes as required by HFEA register.

HABSelect has obtained full approval from NHS Research Ethics Committee (Ref 13/YH/0162) that covers couples undergoing fully funded treatment as well as the majority of private patients in the participating sites. It is being conducted in accordance with good clinical practice principles, Declaration of Helsinki (1996) and Research Governance Framework (2005). It has also been endorsed by the major charity Infertility Network UK, where support and advice via patient participation will be sought on a regular basis. The trial is registered with an authorised registry (ISRCTN99214271) according to the ICMJE Guidelines (http://www.icmje.org), and the authorship credit will be on the substantial contributions as per the same guidelines.

The Trial Steering Committee will agree a publication plan and be consulted prior to release or otherwise publish any study data. We anticipate that in addition to the interim final report required by the funder in Sept 2017 (open access), all outcomes from the study will be submitted for peer review in the appropriate, open access journals. Communications will also be delivered at key international meetings associated with relevant reproductive societies and groupings. Patients and other stakeholders will also be able to obtain information on their arm allocation after accessing a web site that will be set up specifically for this purpose. As per Funder's requirements, all materials to be submitted for publication will be sent to the NIHR Coordinating Centre for EME (NCCEMEM) for approval and prior to publication.

\section{Consent to the BioBank repository}

Couples who are eligible to take part in the trial will also be eligible to have their residual sperm samples stored for future research in the University of Birmingham's established tissue bank called the Human Biomaterials Resource Centre (HBRC), which collects and stores human tissue samples for medical research. Participation will be discussed with patients at the same time as discussing their participation in the main trial. Patients who agree to have residual sperm samples stored will be asked to sign an additional consent form.

\section{DISCUSSION AND CONCLUSIONS}

HABSelect will be the largest male infertility trial undertaken to date in the UK. Like most other studies, it has strengths and limitations (see above). However, its capability to address a significant unmet health need and also advance mechanistic understanding and impact of DNA integrity/fragmentation on clinical success in ICSI 
cannot be understated. In addition to HA-binding, alternative strategies to identify and select sperm for ICSI include biophysical and morphometric methods based on passive and active microfluidic chambers, zeta potential, high resolution imaging (IMSI and IMSOMI) and magnetic cell sorting (MACS). Recent systematic reviews with meta-analyses, however, found little evidence of efficacy with the caveat that all studies were either inadequately powered or were of low quality. ${ }^{29} 4445$ The most recent review of the literature on the efficacy with HA-selection also reported a lack of efficacy but with the same caveat applied. ${ }^{28}$

It may be difficult to exclude sperm with such genotoxic DNA fragmentation altogether from ICSI procedures; it is surely within our means, however, to sufficiently eliminate them from the pool of those prepared for ICSI. The HABSelect trial seeks to provide robust evidence to firmly accept or reject the recommendation of a prior HA-binding step in the selection of sperm for ICSI and to determine whether such selection does indeed mitigate for higher genotoxic potential in patient samples. The study complies fully with and extends on the NICE call for fertility guidance: (http://www.nice.org.uk/newsroom/pressreleases/ NICEOutlinesReviewOfFertilityGuideline.jsp) and the ESHRE call for new markers of sperm quality (https:// www.eshre.eu/ /media/sitecore-files/.../2012-January. pdf?la=en).

It is difficult to assess what impact the PICSI intervention might have on the overall cost of treatment. However, depending on the study outcomes, costeffectiveness modelling alongside an individual patient data (IPD) meta-analysis may be considered.

\section{Trial status}

The first patient was enrolled into HABSelect in December 2013, and recruitment is due to end in August 2016. The study is being conducted in 15 centres across the UK. The trial report should be available in the Autumn of 2017.

\section{CONFLICTS OF INTEREST}

The choice of the PICSI dish for all interventions was based on its ready availability, solid construction, careful quality control and relative ease of use. There were no commercial considerations in its adoption. A successful conclusion of the study could help establish a more consistent and objective procedure for sperm selection by ICSI that can be extended to different HA-selection platforms.

\footnotetext{
Author affiliations

${ }^{1}$ Department: Centre for Primary Care \& Public Health, Queen Mary University of London, London, UK

${ }^{2}$ School of Medicine and Dentistry, University of Aberdeen, Aberdeen, UK

${ }^{3}$ Charity Registration No. 1099960 (InfertilityNetworkUK), London, UK

${ }^{4}$ Centre for Human Reproductive Science, University of Birmingham,

Birmingham Women's Fertility Centre, Birmingham Women's NHS Foundation
}

Trust, Birmingham, UK

${ }^{5}$ Assisted Conception Unit, Guy's and St Thomas's Hospital, London, UK

${ }^{6}$ Queen's University Belfast, Institute of Pathology, Belfast, UK

${ }^{7}$ Department of Human Metabolism, University of Sheffield, Sheffield, UK

${ }^{8}$ Dental Translational and Clinical Research Unit, School of Dentistry,

University of Leeds, Leeds, UK

${ }^{9}$ Leeds Institute of Health Sciences, University of Leeds, Leeds, UK

${ }^{10}$ Leeds Institute of Cardiovascular and Metabolic Medicine (LICAMM),

University of Leeds, Leeds, UK

Twitter Follow Allan Pacey at @allanpacey

Contributors KDW and DM designed and wrote the protocol. SP provided expert assistance on trial design and management. JK-B, SEL and AP provided expert assistance on the application of laboratory methods. $\mathrm{RH}$ and RW provided essential statistical support on clinical and mechanistic aspects of the study, respectively. YK, AC and SB provided expert clinical support and checked the protocol for accuracy. LB designed the clinical statistical analysis plan. KB is our Patient \& Public Involvement Contributor.

Funding This project is funded by the Efficacy and Mechanism Evaluation (EME) Programme, an MRC and NIHR partnership. This work is supported by the UK National Institute for Health Research (ISRCTN No. 99214271; MREC No. 13/YH/0162; UKCRN ID 14845).

Competing interests The authors declare that they have no competing interests.

Disclaimer The views expressed in this publication are those of the author(s) and not necessarily those of the MRC, NHS, NIHR or the Department of Health.

Patient consent Obtained.

Ethics approval IRAS.

Provenance and peer review Not commissioned; externally peer reviewed.

Open Access This is an Open Access article distributed in accordance with the Creative Commons Attribution Non Commercial (CC BY-NC 4.0) license, which permits others to distribute, remix, adapt, build upon this work noncommercially, and license their derivative works on different terms, provided the original work is properly cited and the use is non-commercial. See: http:// creativecommons.org/licenses/by-nc/4.0/

\section{REFERENCES}

1. HFEA. A long term analysis of the HFEA Register data, 1991-2006. http://www.hfea.gov.uk/docs/Latest_long_term_data_analysis_ report_91-06.pdf.pdf

2. HFEA. Latest UK fertility treatment data and figures: 2012-2013. 2016. http://www.hfea.gov.uk/docs/HFEA_Fertility_Trends_and_ Figures_2013.pdf

3. Jungwirth A, Giwercman A, Tournaye $\mathrm{H}$, et al. European Association of Urology guidelines on Male Infertility: the 2012 update. Eur Urol 2012;62:324-32.

4. WHO. WHO laboratory manual for the examination and processing of human semen. WHO, 2010.

5. Gu LJ, Chen ZW, Chen ZJ, et al. Sperm chromatin anomalies have an adverse effect on the outcome of conventional in vitro fertilization a study with strictly controlled external factors. Fertil Steril 2009;92:1344-6.

6. Lewis SE, Aitken RJ. DNA damage to spermatozoa has impacts on fertilization and pregnancy. Cell Tissue Res 2005;322:33-41.

7. Miciński P, Pawlicki K, Wielgus E, et al. The sperm chromatin structure assay (SCSA) as prognostic factor in IVF/ICSI program. Reprod Biol 2009;9:65-70.

8. Simon L, Brunborg G, Stevenson M, et al. Clinical significance of sperm DNA damage in assisted reproduction outcome. Hum Reprod 2010;25:1594-608.

9. Sousa AP, Tavares RS, Velez de la Calle JF, et al. Dual use of Diff-Quik-like stains for the simultaneous evaluation of human sperm morphology and chromatin status. Hum Reprod 2009;24:28-36.

10. Zini A, Libman J. Sperm DNA damage: clinical significance in the era of assisted reproduction. CMAJ 2006;175:495-500.

11. Robinson L, Gallos ID, Conner SJ, et al. The effect of sperm DNA fragmentation on miscarriage rates: a systematic review and meta-analysis. Hum Reprod 2012;27:2908-17. 
12. Zhao J, Zhang Q, Wang $\mathrm{Y}$, et al. Whether sperm deoxyribonucleic acid fragmentation has an effect on pregnancy and miscarriage after in vitro fertilization/intracytoplasmic sperm injection: a systematic review and meta-analysis. Fertil Steril 2014:102:998-1005 e8.

13. Ashwood-Smith MJ, Edwards RG. DNA repair by oocytes. Mol Hum Reprod 1996;2:46-51.

14. Fulka $\mathrm{H}$, Langerova A, Barnetova I, et al. How to repair the oocyte and zygote? J Reprod Dev 2009;55:583-7.

15. Hirst R, Gosden R, Miller D. The cyclin-like uracil DNA glycosylase (UDG) of murine oocytes and its relationship to human and chimpanzee homologues. Gene 2006;375:95-102.

16. Jaroudi S, SenGupta S. DNA repair in mammalian embryos. Mutat Res 2007:635:53-77.

17. Simon L, Lutton D, McManus J, et al. Sperm DNA damage measured by the alkaline Comet assay as an independent predictor of male infertility and in vitro fertilization success. Fertil Steril 2011;95:652-7.

18. Lewis SE, Simon L. Clinical implications of sperm DNA damage. Hum Fertil (Camb) 2010;13:201-7.

19. Dandekar P, Aggeler J, Talbot P. Structure, distribution and composition of the extracellular matrix of human oocytes and cumulus masses. Hum Reprod 1992;7:391-8.

20. Martin-Deleon PA. Germ-cell hyaluronidases: their roles in sperm function. Int J Androl 2011;34:e306-18.

21. Modelski MJ, Menlah G, Wang $\mathrm{Y}$, et al. Hyaluronidase 2: a novel germ cell hyaluronidase with epididymal expression and functional roles in mammalian sperm. Biol Reprod 2014;91:109.

22. Moretti E, Gergely A, Zeyneloglu HB, et al. Relationship among head size, morphology and chromosome structure in human spermatozoa. Fertil Steril 1997;68:138

23. Prinosilova P, Kruger T, Sati L, et al. Selectivity of hyaluronic acid binding for spermatozoa with normal Tygerberg strict morphology. Reprod Biomed Online 2009;18:177-83.

24. Sati L, Ovari L, Bennett D, et al. Double probing of human spermatozoa for persistent histones, surplus cytoplasm apoptosis and DNA fragmentation. Reprod Biomed Online 2008;16:570-9.

25. Parmegiani L, Cognigni GE, Bernardi S, et al. "Physiologic ICSI": hyaluronic acid (HA) favors selection of spermatozoa without DNA fragmentation and with normal nucleus, resulting in improvement of embryo quality. Fertil Steril 2010;93:598-604.

26. Worrilow KC, Eid S, Woodhouse D, et al. Use of hyaluronan in the selection of sperm for intracytoplasmic sperm injection (ICSI): significant improvement in clinical outcomes-multicenter, double-blinded and randomized controlled trial. Hum Reprod 2013;28:306-14.

27. Mokánszki A, Tóthné EV, Bodnár B, et al. Is sperm hyaluronic acid binding ability predictive for clinical success of intracytoplasmic sperm injection: PICSI vs ICSI? Syst Biol Reprod Med 2014:60:348-54.

28. Beck-Fruchter R, Shalev E, Weiss A. Clinical benefit using sperm hyaluronic acid binding technique in ICSI cycles: a systematic review and meta-analysis. Reprod Biomed Online 2016;32:286-98.
29. McDowell S, Kroon B, Ford E, et al. Advanced sperm selection techniques for assisted reproduction. Cochrane Database Syst Rev 2014;10:CD010461.

30. Parmegiani L, Cognigni GE, Bernardi S, et al. Comparison of two ready-to-use systems designed for sperm-hyaluronic acid binding selection before intracytoplasmic sperm injection: PICSI vs Sperm Slow: a prospective, randomized trial. Fertil Steril 2012;98:632-7.

31. Delbes G, Herrero MB, Troeung ET, et al. The use of complimentary assays to evaluate the enrichment of human sperm quality in asthenoteratozoospermic and teratozoospermic samples processed with Annexin- $\mathrm{V}$ magnetic activated cell sorting. Andrology 2013;1:698-706.

32. O'Flaherty C, Vaisheva F, Hales BF, et al. Characterization of sperm chromatin quality in testicular cancer and Hodgkin's lymphoma patients prior to chemotherapy. Hum Reprod 2008;23:1044-52.

33. Enciso M, Sarasa J, Agarwal A, et al. A two-tailed Comet assay for assessing DNA damage in spermatozoa. Reprod Biomed Online 2009:18:609-16.

34. Nijs M, Creemers E, Cox A, et al. Chromomycin A3 staining, sperm chromatin structure assay and hyaluronic acid binding assay as predictors for assisted reproductive outcome. Reprod Biomed Online 2009;19:671-84.

35. Yagci A, Murk W, Stronk J, et al. Spermatozoa bound to solid state hyaluronic acid show chromatin structure with high DNA chain integrity: an acridine orange fluorescence study. $J$ Androl 2010;31:566-72.

36. Zini A, Zhang X, San Gabriel M. Sperm nuclear histone H2B: correlation with sperm DNA denaturation and DNA stainability. Asian $J$ Androl 2008;10:865-71.

37. White IR, Horton NJ, Carpenter J, et al. Strategy for intention to treat analysis in randomised trials with missing outcome data. BMJ 2011;342:d40.

38. Siddique J, Brown $\mathrm{CH}$, Hedeker $\mathrm{D}$, et al. Missing data in longitudinal trials-part B, analytic issues. Psychiatr Ann 2008;38:793-801.

39. Bollen K. Structural equations with latent variables. New York: John Wiley, 1989

40. Wright S. Correlation and causation. J Agric Res 1921;20:557-85.

41. Parmegiani L, Cognigni GE, Ciampaglia W, et al. Efficiency of hyaluronic acid (HA) sperm selection. J Assist Reprod Genet 2010;27:13-16.

42. Peduzzi P, Concato J, Kemper E, et al. A simulation study of the number of events per variable in logistic regression analysis. $J$ Clin Epidemiol 1996;49:1373-9.

43. Nelson SM, Lawlor DA. Predicting live birth, preterm delivery, and low birth weight in infants born from in vitro fertilisation: a prospective study of 144,018 treatment cycles. PLoS Med 2011;8: e1000386.

44. Osman A, Alsomait $\mathrm{H}$, Seshadri S, et al. The effect of sperm DNA fragmentation on live birth rate after IVF or ICSI: a systematic review and meta-analysis. Reprod Biomed Online 2015;30:120-7.

45. Said TM, Land JA. Effects of advanced selection methods on sperm quality and ART outcome: a systematic review. Hum Reprod Update 2011;17:719-33. 
Correction: Hyaluronic Acid Binding Sperm Selection for assisted reproduction treatment (HABSelect): study protocol for a multicentre randomised controlled trial

Witt KD, Beresford L, Bhattacharya S, et al. Hyaluronic Acid Binding Sperm Selection for assisted reproduction treatment (HABSelect): study protocol for a multicentre randomised controlled trial. BMJ Open 2016;6:e012609. doi: 10.1136/ bmjopen-2016-012609

An additional author should appear in the article: Rachel Cutting. Her correspondence details are "Assisted Conception Unit, Jessop Wing, Royal Hallamshire Hospital, Tree Root Walk, Sheffield, S10 2SF, UK; rachel.cutting@sth.nhs.uk”.

The updated Contributors statement should read:

Contributors Witt and Miller designed and wrote the protocol. Pavitt provided expert assistance on trial design and management. Kirkman-Brown, Lewis and Pacey provided expert assistance on the application of laboratory methods. Hooper and West provided essential statistical support on clinical and mechanistic aspects of the study, respectively. Cutting provided essential support with the clinical embryology embedded in the protocol. Khalaf, Coomarasamy and Bhattacharaya provided expert clinical support and checked the protocol for accuracy. Beresford designed the clinical Statistical Analysis Plan. Kate Brian is our Patient \& Public Involvement Contributor.

Open Access This is an Open Access article distributed in accordance with the Creative Commons Attribution Non Commercial (CC BY-NC 4.0) license, which permits others to distribute, remix, adapt, build upon this work non-commercially, and license their derivative works on different terms, provided the original work is properly cited and the use is non-commercial. See: http://creativecommons.org/licenses/by-nc/4.0/

C Article author(s) (or their employer(s) unless otherwise stated in the text of the article) 2017. All rights reserved. No commercial use is permitted unless otherwise expressly granted.

BMJ Open 2017;7:e012609corr1. doi:10.1136/bmjopen-2016-012609corr1

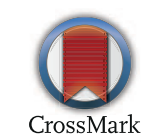

